\title{
What the heart forgets: Cardiac timing influences memory for words and is modulated by metacognition and interoceptive sensitivity
} \author{
ANIL K. SETH, ${ }^{\mathrm{b}, \mathrm{c}}$ AND HUGO D. CRITCHLEY, ${ }^{\mathrm{a}, \mathrm{b}}$ \\ ${ }^{a}$ Department of Psychiatry, Brighton and Sussex Medical School, University of Sussex, Falmer, UK

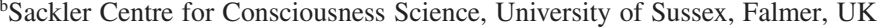 \\ 'Department of Informatics, University of Sussex, Falmer, UK \\ ${ }^{\mathrm{d}}$ U.O. Direzione Scientifica, Fondazione IRCCS Istituto Neurologico Carlo Besta, Milano, Italy \\ 'Department of Neurology, Brighton and Sussex Medical School, University of Sussex, Falmer, UK \\ ${ }^{\mathrm{f}}$ Wellcome Trust Centre for Neuroimaging, University College London, London, UK
}

SARAH N. GARFINKEL, ${ }^{\mathrm{a}, \mathrm{b}}$ ADAM B. BARRETT, ${ }^{\mathrm{b}, \mathrm{c}}$ LUDOVICO MINATI, ${ }^{\mathrm{a}, \mathrm{de} e}$ RAYMOND J. DOLAN ${ }^{\mathrm{f}}$

\begin{abstract}
Mental functions are influenced by states of physiological arousal. Afferent neural activity from arterial baroreceptors at systole conveys the strength and timing of individual heartbeats to the brain. We presented words under limited attentional resources time-locked to different phases of the cardiac cycle, to test a hypothesis that natural baroreceptor stimulation influences detection and subsequent memory of words. We show memory for words presented around systole was decreased relative to words at diastole. The deleterious memory effect of systole was greater for words detected with low confidence and amplified in individuals with low interoceptive sensitivity, as indexed using a heartbeat counting task. Our observations highlight an important cardiovascular channel through which autonomic arousal impacts a cognitive function, an effect mitigated by metacognition (perceptual confidence) and interoceptive sensitivity.
\end{abstract}

Descriptors: Learning/Memory, ECG/EKG, Cognition, Interoception, Baroreceptor

Bodily states of arousal can influence both emotional experience and the way in which stimuli are processed. Body-mind/brain interactions are perhaps most relevant to the understanding of affective processes, including emotional experience. James (1884) and Lange (1885) postulated a causal relationship between body and mind, suggesting that physiological responses within the body provide emotional color and warmth to sensory perceptions: "Without the bodily states following on the perception, the latter would be purely cognitive in form, pale, colorless, destitute of emotional warmth" (James, 1890/1950, pp. 449-450). The integration of physiological states of arousal with perceptual and cognitive processing is also embedded within subsequent theoretical models of emotion, for example, the two-stage model (Schachter \& Singer, 1962) and the Somatic Marker Hypothesis (Bechara, Damasio, Damasio, \& Lee, 1999; Damasio, 1996; Damasio, Tranel, \& Damasio, 1990). There is now an emerging knowledge regarding

This work was supported by a donation from the Dr. Mortimer and Theresa Sackler Foundation. AKS is also supported by EU Project CEEDS FP7-ICT-2009-05, 258749, and an Engineering and Physical Sciences Research Council Leadership Fellowship EP/G007543/1, which also supports the work of ABB. Many thanks to Dr. Ayana Gibbs who provided advice on EAB design and Ewan Leith who assisted with stimulus selection.

Address correspondence to: Sarah N. Garfinkel, Clinical Imaging Science Centre, Brighton and Sussex Medical School, University of Sussex, Falmer BN1 9RR, UK. E-mail: s.garfinkel@bsms.ac.uk the neural mechanisms and interoceptive pathways that support these interactions (Critchley, Wiens, Rotshtein, Ohman, \& Dolan, 2004; Harrison, Brydon, Walker, Gray, Steptoe, \& Critchley, 2009; Harrison, Brydon, Walker, Gray, Steptoe, Dolan, et al., 2009; Levinthal \& Strick, 2012).

Memory is affected by states of cognitive and physiological arousal. Arousing material is better remembered than nonarousing material, leading to an advantage for subsequent memory (Cahill \& McGaugh, 1998; Dolcos \& Cabeza, 2002; Kensinger \& Corkin, 2003), a process that is mediated though monoamineenhanced amygdalohippocampal coupling (McGaugh, Cahill, \& Roozendaal, 1996). Physiologically, heart rate and electrodermal indices of sympathetic arousal correlate with subsequent memory (Bradley, Greenwald, Petry, \& Lang, 1992). Functional perturbation of neural pathways that control and represent changes in internal bodily arousal also impact on memory for both emotional and neutral material (Clark, Naritoku, Smith, Browning, \& Jensen, 1999; Critchley et al., 2007; Heims et al., 2006; Nogueira, Tomaz, \& Williams, 1994; Williams \& McGaugh, 1993). Together, these findings reinforce wider evidence that interoceptive information can influence memory formation.

One major route conveying information about bodily arousal is baroreceptor firing. Baroreceptors are pressure and stretch receptors within the major arteries from the heart (aortic arch and carotid bodies) that fire in bursts every time the heart empties at systole and are silent between heartbeats during diastole. These signals are 
carried by vagus and glossopharyngeal nerve afferents to the nucleus of the solitary tract and encode the timing and strength of individual heartbeats. This information is critical for control of blood pressure (via a brainstem baroreflex), and there is evidence that it also impacts on perceptual and cognitive processing. Thus, cardiac timing influences the processing of unexpected somatosensory stimuli, pain, and emotional faces (Donadio, Karlsson, Elam, \& Wallin, 2002; Gray et al., 2012; Gray, Minati, Paoletti, \& Critchley, 2010; Gray, Rylander, Harrison, Wallin, \& Critchley, 2009). Brain regions mediating these effects include the dorsal and periaqueductal gray matter, amygdala, insula, and orbitofrontal cortex (Gray et al., 2009, 2012). Behavioral and neuroimaging experiments using cardiac timing therefore give precision in understanding mechanisms through which physiological arousal states influence mental processes by tapping into a specific neural channel of interoceptive information.

In principle, if this channel of interoceptive information is important for mediating influences of physiological arousal (notably heart rate and blood pressure increases) on perceptual, cognitive, and emotional functions, then this may be reflected in the differential processing of brief stimuli presented at different times in the cardiac cycle. Stimuli presented at systole will coincide with central representation of the afferent baroreceptor discharge, while stimuli presented at diastole will not.

The extent to which physiological changes influence cognitive processes may reflect the magnitude and intensity of the peripheral arousal, but also individual differences in the quality of viscerosensory representation within the brain. People vary in their sensitivity to internal bodily states (interoceptive sensitivity). While interoceptive sensitivity is a presumed stable trait across individuals (Ehlers \& Breuer, 1992; Ehlers, Breuer, Dohn, \& Fiegenbaum, 1995; Khalsa et al., 2008), heartbeat detection can be altered on a within-subjects basis through variations in current emotional state (e.g., state anxiety; Ludwickrosenthal \& Neufeld, 1985; Paulus \& Stein, 2010), and by manipulating factors such as posture (Schandry, Bestler, \& Montoya, 1993) and physical load (Schandry et al., 1993). Interoceptive sensitivity is linked to differences in emotional behavior and reported emotional experiences (Cameron, 2001; Domschke, Stevens, Pfleiderer, \& Gerlach, 2010; Herbert, Herbert, \& Pollatos, 2011; Herbert, Pollatos, Flor, Enck, \& Schandry, 2010; Katkin, 1985; Vaitl, 1996; Wiens, Mezzacappa, \& Katkin, 2000). Heartbeat detection and/or timing tasks are objective means for testing the degree of interoceptive sensitivity, predicated on the notion that the accuracy with which people can perceive their heart beating when at rest reflects more general sensitivity to interoceptive signals. Individuals that score high on interoceptive sensitivity are better able to utilize bodily signals to guide decision making (Damasio, 1996; Dunn et al., 2010; Werner, Jung, Duschek, \& Schandry, 2009), subliminal learning (Katkin, Wiens, \& Ohman, 2001), and implicit memory (Werner, Peres, Duschek, \& Schandry, 2010), while being less susceptible to illusions of body ownership (Tsakiris, Tajadura-Jimenez, \& Costantini, 2011).

Uniting these strands of research, we investigated the impact of afferent cardiovascular information and interoceptive sensitivity on attentional and memory processes. We first tested for the influence of cardiac timing on the detection of emotional and neutral word stimuli under limited attentional resources, using a modified emotional attentional blink (EAB) paradigm (Anderson, 2005; Anderson \& Phelps, 2001; Keil \& Ihssen, 2004). Here, when a participant is required to identify two stimuli in a stream of stimuli (rapid serial visual presentation; RSVP), the identification of a second target (T2) is typically obscured (masked) by the detection of a first target (T1) around $350 \mathrm{~ms}$ earlier, such that attention "blinks" after the detection of the T1 stimulus (and misses the T2 stimulus). If T2 is an emotional stimulus (e.g., a negative word such as "murder;" Anderson, 2005, or a fear face; De Martino, Kalisch, Rees \& Dolan, 2009), there is more likelihood that it will be detected, suggesting "breakthrough" of the attentional blink by virtue of its salience (Anderson \& Phelps, 2001; Keil \& Ihssen, 2004). In the present study, we used word stimuli within an EAB task that replicated the established task design (Anderson \& Phelps, 2001; Keil \& Ihssen, 2004), but we also time-locked T2 presentation to coincide with either diastole or systole. We tested participants behaviorally for the detection of T1 and T2 stimuli using forced choice tasks and also measured (metacognitive) confidence in their detection judgment. Participants were later tested for memory (free recall) for T2 items seen in the attentional blink task. Thus, we specifically tested whether the baroreceptor channel of interoceptive information alters attention-dependent processing of emotional words (i.e., their detection) and whether it impacts subsequent memory for these words. We first hypothesized an inhibitory or competitive effect of baroreceptor firing on processing of word stimuli (Vaitl, 1996) that would manifest as a deleterious effect on the detection and memory for stimuli presented at cardiac systole. Moreover, we anticipated that this effect would interact with individual differences in interoceptive sensitivity, predicting that people with high scores on heartbeat detection/timing tasks would show the greatest influence of heart timing on detection and memory processes.

\section{Method}

\section{Participants}

The study was approved by a local research ethics committee. Twenty-two healthy participants were recruited from advertisements placed at the University of Sussex and on community websites in Brighton, England. All participants underwent an EAB paradigm (14 females, 8 males). Two participants were excluded (one due to equipment failure, the other had a heart rate of over 115 beats per minute; mean $\pm S D$ of others, $74.27 \pm 9.59$ beats per minute). An additional three participants did not sit the memory test. All analyses were performed on $N=17$ participants (12 females, 5 males), mean age $26.7 \pm 8.58$ years.

\section{Procedure}

To investigate potential cardiac modulation of attention, an established EAB paradigm (Anderson \& Phelps, 2001) was followed. Trials involved the RSVP of 15 words. Words were presented at high speed $(100 \mathrm{~ms})$ and in white on a black background with the exception of two targets (T1 \& T2), which were presented in green. Participants were instructed to identify both T1 and T2 stimuli, and the primary function of T1 was to mask T2. All T1s were neutral $(N=210)$. T2s included negative $(n=50)$, positive $(n=50)$, and neutral $(n=110)$ items. The task was time-locked to the electrocardiograph (ECG): Half of all T2 items were time-locked to the R wave (during cardiac diastole) and half to the $\mathrm{T}$ wave (occurring at cardiac systole, synchronous with baroreceptor discharge) (Figure 1). After each RSVP, participants were instructed to type into the computer the $\mathrm{T} 1$ for that trial, followed by their confidence on a 1-5-point scale. They were then prompted to type in T2, followed by their confidence judgment (again on a 1-5 scale). All 


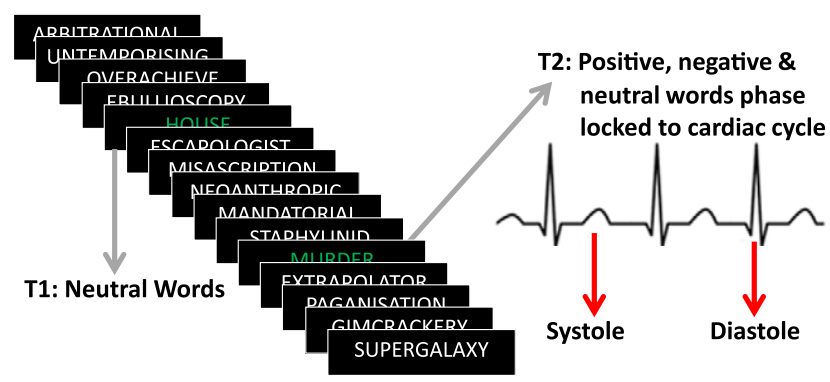

Figure 1. Emotional attentional blink task with cardiac timing. Each RSVP consisted of 15 items, 13 were long distractor words presented in white, and 2 were target words ( $\mathrm{T} 1$ and $\mathrm{T} 2$ ) presented in green. $\mathrm{T} 1$ was a neutral word, and was always presented first in order to enhance the masking of T2. T2 was the stimulus of interest, and was either a neutral (e.g., fence), positive (e.g., happy), or negative (e.g., murder) word. T2 was time-locked to either the $\mathrm{R}$ wave (diastole) or $\mathrm{T}$ wave (systole). Detection of $\mathrm{T} 2$ served as the variable of interest for attention (as determined on a trial-by-trial basis). Memory for T2 items was assessed at the end of the experiment using free recall.

correct detections were scored ( 1 for correct, 0 for incorrect) for all T2 stimuli (stimuli of interest as the masked stimulus time-locked to cardiac cycle), serving as our measure for attention.

Memory. After a delay of approximately $1 \mathrm{~h}$, participants were given a surprise free recall test. They were asked to write down all target words they could remember from the EAB procedure. Participants were given a maximum of $10 \mathrm{~min}$ to complete this free recall test.

Interoceptive sensitivity. Interoceptive sensitivity was assessed using the mental tracking task of Katkin, Morell, Goldband, Bernstein, and Wise (1982). In the mental tracking task, participants were given verbal instructions: "Without manually checking, can you silently count each heartbeat you feel in your body from the time you hear 'start' to when you hear 'stop.' " The task was repeated six times, using randomly presented time windows of 25, 30, 35, 40, 45, and $50 \mathrm{~s}$. Accuracy score was computed for each trial:

$$
1-\frac{\mid \text { nbeats }_{\text {real }}-\text { nbeat }_{\text {reported }} \mid}{\left(\text { nbeats }_{\text {real }}+\text { nbeats }_{\text {reported }}\right) / 2}
$$

These accuracy scores were averaged over the six presentations, yielding an average interoceptive score for each participant.

\section{Data Analysis}

Attention. For the attentional blink task, we conducted a repeated measures $2 \times 3$ analysis of variance (ANOVA) with cardiac cycle (systole, diastole) and valence (negative, positive, neutral) of T2 words as the independent variables, and percentage of words detected (attentional breakthrough) as the dependent variable.

Memory. Memory effects were determined using memory scores corrected for baseline rates of breakthrough detection during the EAB task. This ensured we were testing for memory of correctly detected stimuli and that memory effects were independent of any potential slight (but nonsignificant) modifications of cardiac cycle on baseline detection rates. We again used a $2 \times 3$ repeated measures ANOVA using cardiac cycle (systole, diastole) and emotion (negative, positive, neutral) as independent within-participant variables, and T2 words recalled (corrected for baseline breakthrough) as the dependent variable. Effects obtained were not significantly altered when using raw memory scores (i.e., number of $\mathrm{T} 2$ words recalled, not adjusted for rates of detection during the attentional blink), as illustrated in Figure 3.

Confidence. Confidence during word detection in the attentional blink, and implications for cardiac modulation of memory, were explored using a $2 \times 2$ repeated measures ANOVA, with memory status (remembered, forgotten) and cardiac cycle (systole, diastole) as independent factors, and confidence (that T2 was correctly detected during the attentional blink) as the dependent variable. Follow-up analyses used paired $t$ tests to investigate differences in the amount of high detection-confidence and low detectionconfidence words recalled as a function of cardiac cycle. As detection confidence was performed on a 5-point scale, high confidence words were defined as those detected with confidence 3-5 and low confidence words as 1-2.

In addition, a receiver operating characteristic (ROC) analysis investigated in greater detail the extent to which T2 confidence during the attentional blink predicted subsequent memory (Macmillan \& Creelman, 2004). An ROC analysis explores the extent to which a signal (here, T2 confidence) is effective as a detector of some binary state variable $S$ (here, subsequent T2 word memory), at all possible detection thresholds. When the signal exceeds the detection threshold, one predicts that $S=1$ (i.e., in this case, that the T2 word will be remembered). For each detection threshold, one computes the hit rate (i.e., proportion of $S=1$ trials successfully detected/predicted as $S=1$ ) and the false alarm rate (proportion of $S=0$ trials wrongly detected/predicted as $S=1$ ). The ROC curve plots the hit rate versus the false alarm rate over all the possible detection thresholds. In this case, there were six possible detection thresholds, since there were five confidence ratings: to predict memory on every trial, to predict memory when confidence was greater than 1 , to predict memory when confidence was greater than 2, to predict memory when confidence was greater than 3 , to predict memory when confidence was greater than 4 , and to never predict memory. The area under the ROC curve gives a robust measure of the extent to which confidence during T2 detection is diagnostic of subsequent memory, independent of the overall

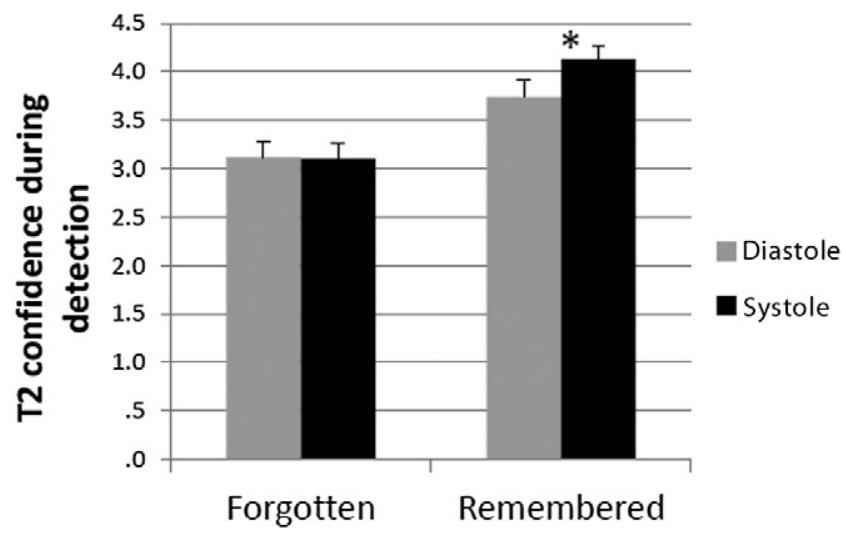

Figure 2. Memory for words processed at systole tended to encompass only those detected with high confidence, whereas words at diastole that were subsequently recalled were associated with both high and low confidence items as reflected in an overall lower mean. 
tendency of the subject to report specific levels of confidence on the 5-point scale. Separate ROC curves were constructed for words presented at systole and those at diastole. Within-subjects $t$ test comparisons on ROC area-under-the-curve measurements were used to identify differences in the extent to which confidence during T2 word detection influenced subsequent word memory on diastole relative to systole.

Interoceptive sensitivity. To explore individual differences in cardiac modulation of memory, interoceptive sensitivity was included in the ANOVA as a covariate of interest, to determine whether cardiac timing during the attentional blink modulated memory differentially based upon individual differences in interoception. In addition, using Pearson's $r$, number of words recalled was correlated with interceptive sensitivity score. Finally, the potential for confidence during word detection to predict subsequent memory at different parts of the cardiac cycle (systole, diastole), as detailed by the ROC analysis, was also correlated with interoceptive sensitivity score.

All significant main effects and interactions, and other nonsignificant effects of potential interest, are accompanied by means and standard deviations (mean $\pm S D$ ). A Greenhouse-Geisser procedure was used in the event that the sphericity assumption was violated in the ANOVAs.

\section{Results}

\section{Attention}

Using our task, we replicated the EAB effect (Anderson, 2005; Anderson \& Phelps, 2001) and the observation that emotional T2 stimuli are more likely to break through the attentional blink (shown as a main effect of emotion for words detected, $F[2,32]=27.46, p<.001$, and separately for percentage of positive, $60.15 \pm 13.91, t[16]=5.69, p<.001$, and negative words, $62.30 \pm 16.47, t[16]=6.29, p<.001$, relative to percentage of neutral words, $48.27 \pm 13.87$ ). There was no difference between breakthrough of negative versus positive words, $t(16)=1.25$, $p=.23$. We did not observe a main effect of cardiac cycle on detection accuracy of percentage of T2 stimuli identified (diastole $57.00 \pm 14.7$ and systole $57.11 \pm 13.80), F(1,16)=0.01, p=.93$, and there was also no emotion by cardiac timing interaction for detection, $F(2,32)=2.07, p=.14$.

Confidence was higher for accurately detected T2 words $(3.91 \pm 0.56)$ relative to those not detected $(1.88 \pm 0.44)$ $F(1,16)=-100.63, p<.001$. Cardiac timing had no effect on the overall confidence with which words were detected (no difference in confidence for words detected at diastole [2.90 \pm 0.48$]$ and systole $[2.91 \pm 0.48], F[1,16]=0.006, p=.941$, and no cardiac cycle by detection accuracy interaction for confidence ratings, $F[1,16]=1.506, p=.237$ ); thus, words were accurately detected to an equivalent extent on systole and diastole and were done so with equal confidence.

\section{Memory}

In contrast to the attention results, we observed a main effect of cardiac cycle on subsequent recollection of T2 stimuli detected during the $\mathrm{EAB}, F(1,15)=12.46, p=.003$, indicating impaired memory for words (percentage recalled) originally detected at systole $(21.3 \pm 15.2)$ relative to those detected at diastole $(26.6 \pm 13.4)$. There was no main effect of emotion on subsequent memory, $F(2,30)=1.774, p=.187$. No interactions involving emotion reached significance $(p>.3)$.

\section{Confidence of T2 Detection as a Function of Cardiac Cycle and Subsequent Memory}

We examined how the confidence with which people reported detecting T2 stimuli related to cardiac timing of the stimuli and subsequent memory (rated on a $0-5$ scale). Higher detection confidence predicted memory (main effect, $F(1,15)=51.58, p<.001$, mean confidence during detection of subsequently remembered, $4.02 \pm 0.61$, and forgotten words, $3.11 \pm 0.64)$, and was significantly influenced by cardiac cycle, $F(1,15)=7.42, p=.016$, such that words presented at systole were detected with higher confidence $(3.68 \pm 0.52)$ relative to diastole $(3.47 \pm 0.64)$. Moreover, there was a significant cardiac cycle by memory interaction, $F(1,15)=5.75, p=.030$; with higher detection confidence ratings for subsequently remembered $\mathrm{T} 2$ items presented at systole $(4.2 \pm 0.59)$ relative to diastole $(3.82 \pm 0.72), t(15)=-2.781$, $p=.014$ (see Figure 2). This finding was driven by memory for an equal percentage of high confidence items (those rated 3-5) detected at both diastole (43.44 \pm 14.79 and systole $(41.61 \pm 9.15), t(16)=0.36, p=.722$, but with a significantly higher percentage of low confidence items (those rated 1-2, $11.68 \pm 11.34$ ) detected at diastole subsequently recalled (relative to percentage of low confidence items rated 1-2 detected at systole, $3.26 \pm 6.49), t(16)=2.66, p=.017$. Thus, the equal number of high confidence items recalled irrespective of cardiac cycle, with the addition of memory for more low confidence items detected at diastole, drove down the mean confidence rating for subsequently recalled words at diastole relative to systole.

This effect was further confirmed by an ROC area-under-thecurve analysis measuring the extent to which perceptual confidence during detection was diagnostic of subsequent word memory. It was found that high confidence was more predictive of subsequent memory for words detected on systole (i.e., greater area under the ROC curve for T2 items detected on systole, $0.70 \pm 0.13$, relative to diastole, $0.63 \pm 0.09, t[15]=-2.238, p=.041)$.

\section{Individual Differences: Interoceptive Sensitivity and Memory}

Individual differences in interoceptive sensitivity, as measured by heartbeat detection task accuracy, influenced the extent to which cardiac timing impacted subsequent memory, interaction $F(1,15)=9.753, p=.007$, such that the deleterious effect of $\mathrm{T} 2$ presentation at systole on subsequent memory was attenuated in participants who scored high on interoceptive sensitivity.

This observation was further explored in correlational analyses: The number of words recalled at diastole versus systole correlated significantly with interoceptive sensitivity score $(r=.628$, $p=.007)$. This effect reflected a significant relationship between the number of words remembered at systole $(r=.613, p=.009)$ and level of interoceptive awareness. Moreover, this tendency for high interoceptive sensitivity to enhance memory for words detected at systole (but not diastole) persisted for all emotion categories (see Figure 3).

An individual difference analysis explored whether variations in interoceptive sensitivity also affected the propensity for only high confidence items detected at systole to be subsequently remembered. Interoceptive sensitivity was not related to areaunder-the-confidence-memory ROC curve when T2 items were 


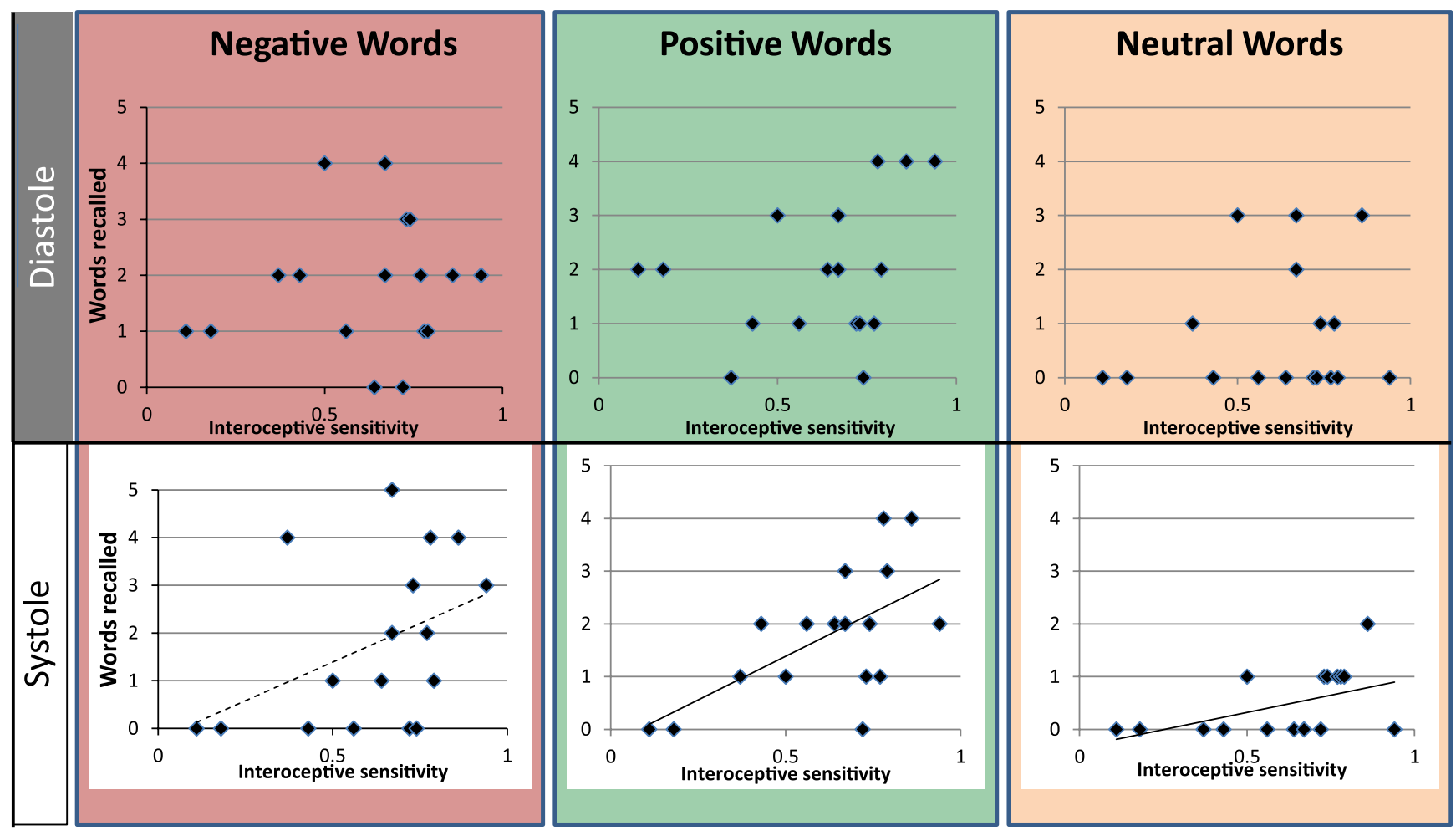

Figure 3. Further analyses explored the relationship between interoceptive sensitivity and cardiac modulation of the subsequent memory for words. Scatter plots depict the relationship between interoceptive sensitivity ( $x$ axis) and number of words recalled ( $y$ axis) for negative, positive, and neutral words. Graphs depicting words originally detected after presentation on diastole are shown in the upper half, with words originally detected after presentation on systole presented in the lower half. Solid trend lines added only to significant correlations, obtained for categories of words originally detected on systole correlating with interoceptive sensitivity (positive words and interoceptive sensitivity on systole $[r=.609, p=.009]$, neutral words and interoceptive sensitivity on systole $[r=.482, p=.050]$ ). Dashed trend line indicates trend relationship between interoceptive sensitivity and memory for negative words originally detected on systole $(r=.429, p=.086)$. No correlations for word valences originally detected on diastole significantly correlated, or trended towards, a relationship with interoceptive sensitivity $(p>.2)$.

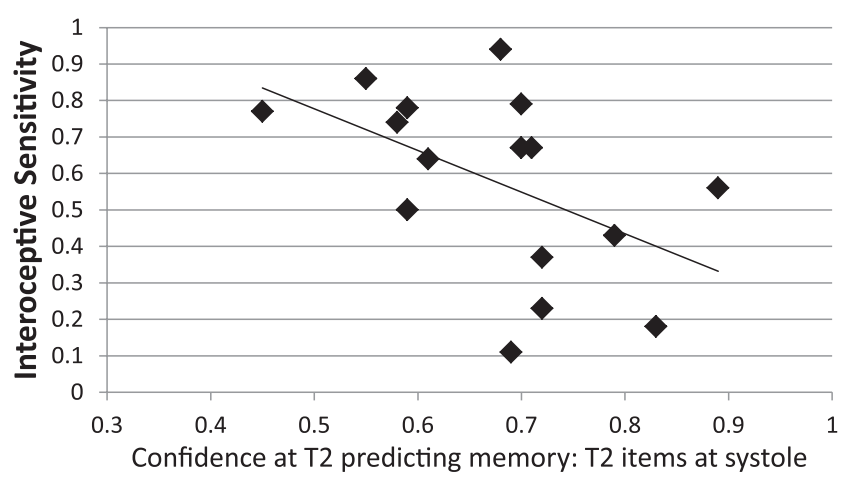

Figure 4. ROC analysis (to predict the degree to which confidence is diagnostic of subsequent memory performance) for $\mathrm{T} 2$ items detected at systole, and inverse correlation with interoceptive sensitivity. Individuals with low interoceptive sensitivity only subsequently remembered words that were identified during EAB with high confidence. Those individuals with high interoceptive sensitivity were less dependent on high confidence during T2 detection at systole to dictate subsequent memory, and had enhanced memory for all subsequent items beyond constraints imposed by confidence encountered by individuals with low interoceptive sensitivity.

detected at diastole $(r=-.206, p=.428)$, whereas heightened interoceptive sensitivity inversely correlated with ROC at systole $(r=-.507, p=.045)$ (see Figure 4). Thus, individuals high in interoceptive sensitivity were able to overcome the constraints of predominantly remembering only items at systole detected with high confidence.

\section{Discussion}

Cardiac cycle influences memory for words: In our experiment using the EAB paradigm, target words detected, and thus encoded, at systole were less well remembered than target words detected at diastole. This cardiac modulation of subsequent memory was influenced by both the degree of confidence with which the words were detected on each trial (metacognition) and by individual differences in interoceptive sensitivity (quantified using a heartbeat detection task). While cardiac cycle did not affect detection breakthrough of target words during the attentional blink paradigm (i.e., attention), those words processed at systole and subsequently remembered tended to be those detected with high confidence. In contrast, words processed at diastole that were subsequently recalled included both high and low confidence items (apparent as reflected in an overall lower mean detection confidence for remembered items). Individuals with heightened interoceptive sensitivity were less susceptible to the deleterious effect of systolic interference on subsequent memory, and consequently showed less dependence on high confidence at initial detection for later recollection. This suggests that the fidelity/reliability of interoceptive representation mitigates the interference of physiological arousal on memory. Together, our 
results suggest that the effectiveness of encoding into memory is influenced by the timing of word stimuli relative to individual heartbeats, an effect that is modulated by attentional processes related to metacognition and individual differences in sensitivity to internal bodily signals, which both protect against visceral interference on cognitive functioning.

Our findings demonstrate an effect of phasic cardiac afferent information, presumed to originate in competitive or inhibitory effects of baroreceptor discharge on the processing of word stimuli to memory. Moreover, we show that these heartbeat timing effects can be modulated independently by the attention-dependent perceptual accuracy of stimulus detection (reflected in metacognitive confidence judgments, rather than first-order detection accuracy) and by individual differences in the accuracy with which participants can judge their internal bodily state. Our findings reveal the interaction of these two mitigating mechanisms, suggesting that people who have more accurate general representation of interoceptive phenomena (such as a better predictive model through fidelity of cardiac feedback; Seth, Suzuki, \& Critchley, 2012) are less distracted by cardiac timing effects during visual perception and hence have a lower dependency on detection confidence to encode words into memory.

Cardiac timing did not affect baseline detection rates of stimuli during the attentional blink, which argues against the memory effects being mediated by assumed general inhibitory properties of baroreceptor activation on low-level stimulus processing or perception under high attentional load (Vaitl, 1996). Thus, the expression of cardiac timing effects on memory is likely to arise during (at least the early phase of) memory consolidation. The central release of monoamines, including those implicated in memory consolidation, is sensitive to afferent baroreceptor signals (Murase, Inui, \& Nosaka, 1994). Thus, the activity of neurons within the locus coeruleus, the major source of noradrenergic projections to amygdala and cortex, is gated by baroreceptor discharge (Elam, Yao, Svensson, \& Thoren, 1984; Murase et al., 1994). Moreover, the firing and refractory properties of locus coeruleus neurons have been proposed to underlie the attentional blink effect (De Martino, Strange, \& Dolan, 2008; Nieuwenhuis, Gilzenrat, Holmes, \& Cohen, 2005). Previous studies using the attentional blink task implicate noradrenaline in attentional breakthrough: Beta-adrenergic blockade (by Propranolol) impairs target detection, while selective enhancement of noradrenaline (with Reboxetine) increases target detection (De Martino et al., 2008). Noradrenergic activity also supports memory consolidation by enhancing interactions between amygdala and hippocampus (McGaugh et al., 1996). Elevated memory for emotionally arousing experiences is associated with heightened levels of noradrenaline (McGaugh \& Roozendaal, 2002), while direct infusion of noradrenaline into basolateral amygdala enhances memory (Liang, Juler, \& McGaugh, 1986; Liang, McGaugh, \& Yao, 1990). Our experimental findings did not include a primary effect of cardiac cycle on target detection, yet it remains plausible that the observed effects on subsequent memory may nevertheless be mediated by ascending monoaminergic (including noradrenergic) pathways.

Individuals with high interoceptive sensitivity did not display systole-associated reductions in memory, suggesting that they are resistant to detrimental effects of baroreceptor activity on memory encoding. This contrasts with a hypothesis from the writings of Lacey and Lacey (1978) that there is a general cortical inhibition of processing that occurs at each cardiac systole and which originates in baroreceptor discharge. While this view suggests that a cardiac timing effect ought to be magnified in individuals with higher interoceptive sensitivity, our results instead suggest a protective, rather than exacerbating, effect on the encoding and consolidation for items detected at systole in individuals with high interoceptive sensitivity. Here, we found that interoceptive sensitivity was associated with increased subsequent memory for all items detected at systole. One interpretation is that (healthy) individuals with enhanced interoceptive sensitivity can generate a high-fidelity predictive model of internal state (Seth et al., 2012), whereby the accurate predictive representation of internal bodily signals results in a focused attribution of baroreceptor traffic. The central effects of afferent baroreceptor inputs are thus attenuated early on by accurate prediction and therefore interact less with stimulus processing. This ultimately weakens the detrimental effect of afferent cardiovascular information on subsequent memory. Alternatively, the mechanism may relate to emotional enhancement of memory. In neuroimaging studies, attention directed towards one's heartbeat activates insula, cingulate, and somatosensory regions (Cameron \& Minoshima, 2002; Critchley et al., 2004; Pollatos, Schandry, Auer, \& Kaufmann, 2007) implicated in subjective feeling of emotion (Critchley, Mathias, \& Dolan, 2001; Damasio et al., 2000). Moreover, individuals with high interoceptive sensitivity report an increased intensity of emotional experiences (Herbert et al., 2011; Wiens et al., 2000). Thus, contextual emotional effects, amplified by interoceptive sensitivity (and perhaps independently of noradrenaline release) may potentiate encoding (Cahill \& McGaugh, 1998) and decrease the detrimental effect of baroreceptor activity on subsequent memory. Further research on the interaction between interoceptive sensitivity and memory is warranted.

Nevertheless, memory effects, while interacting with cardiac cycle, were not modulated by the emotionality of the material. This is consistent with observed effects of stress on EAB, where T2 detection is increased during stress irrespective of T2 valence (Schwabe \& Wolf, 2010). Here, participants who react to stress with a large cortisol response showed less of an attentional blink effect relative to low cortisol responders.

Cardiac modulation effects were not observed for measures of attention, as no significant difference in breakthrough detection rates or confidence ratings were obtained for targets displayed on systole relative to diastole. While it is possible that this interoceptive channel is most sensitive to consolidation-based memory effects, it is nevertheless surprising that attention, which is also sensitive to noradrenergic modulation, was not altered as a function of cardiac cycle. One possible explanation is that this channel of information is sensitive to emotion and that our word emotion categories were not sufficiently strong for an effect to be elicited. The use of more potently emotional stimuli (such as emotional faces) may increase signal. Moreover, our word categories were defined by valence (positive, neutral, and negative), and collapsed across emotions (e.g., our negative category encompassed disgust, fear, and anger words). Orientating heart rate response is distinct for different emotions (Critchley et al., 2005), so it is thus conceivable that cardiac modulation effects on attention are selective for different emotions. Collapsing across emotions could have masked any potential emotion-specific effects of cardiac modulation, thus serving as a limitation of this study. Our statistical significance was, at times, weak, and although multiple tests were used to garner support for shared hypotheses, there remains some risk of Type I error. Future experiments should further confirm findings and should investigate separately cardiac modulation effects on the detection and memory of distinct emotion categories. Moreover, 
studies investigating individual differences typically require a high number of participants, and future experiments should increase sample size to further delineate the effect of trait-level differences in interoceptive sensitivity on cardiac modulation of attention and memory.

Together, our findings demonstrate how a major interoceptive channel that conveys information about cardiovascular arousal impacts on memory. We show distinct mitigating roles of enhanced perceptual representation (indexed by metacognitive access) and enhanced interoceptive sensitivity on the relative deficits in recalling items presented at systole. These findings have implications for understanding the role of visceral state and interoceptive sensitivity on aspects of affective cognition and behavior.

\section{References}

Anderson, A. K. (2005). Affective influences on the attentional dynamics supporting awareness. Journal of Experimental Psychology-General, $134,258-281$

Anderson, A. K., \& Phelps, E. A. (2001). Lesions of the human amygdala impair enhanced perception of emotionally salient events. Nature, 411, 305-309.

Bechara, A., Damasio, H., Damasio, A. R., \& Lee, G. P. (1999). Different contributions of the human amygdala and ventromedial prefrontal cortex to decision-making. Journal of Neuroscience, 19, 54735481.

Bradley, M. M., Greenwald, M. K., Petry, M. C., \& Lang, P. J. (1992). Remembering pictures: Pleasure and arousal in memory. Journal of Experimental Psychology: Learning, Memory, and Cognition, 18, 379390.

Cahill, L., \& McGaugh, J. L. (1998). Mechanisms of emotional arousal and lasting declarative memory. Trends in Neuroscience, 21, 294-299.

Cameron, O. G. (2001). Interoception: The inside story-A model for psychosomatic processes. Psychosomatic Medicine, 63, 697-710.

Cameron, O. G., \& Minoshima, S. (2002). Regional brain activation due to pharmacologically induced adrenergic interoceptive stimulation in humans. Psychosomatic Medicine, 64, 851-861.

Clark, K. B., Naritoku, D. K., Smith, D. C., Browning, R. A., \& Jensen, R. A. (1999). Enhanced recognition memory following vagus nerve stimulation in human subjects. Nature Neuroscience, 2, 94-98.

Critchley, H. D., Lewis, P. A., Orth, M., Josephs, O., Deichmann, R., Trimble, M. R., \& Dolan, R. J. (2007). Vagus nerve stimulation for treatment-resistant depression: Behavioral and neural effects on encoding negative material. Psychosomatic Medicine, 69, 17-22.

Critchley, H. D., Mathias, C. T., \& Dolan, R. J. (2001). Neuroanatomical basis for first- and second-order representations of bodily states. Nature Neuroscience, 4, 207-212.

Critchley, H. D., Rotshtein, P., Nagai, Y., O’Doherty, J., Mathias, C. J., \& Dolan, R. J. (2005). Activity in the human brain predicting differential heart rate responses to emotional facial expressions. Neuroimage, 24, 751-762.

Critchley, H. D., Wiens, S., Rotshtein, P., Ohman, A., \& Dolan, R. J. (2004). Neural systems supporting interoceptive awareness. Nature Neuroscience, 7, 189-195.

Damasio, A. R. (1996). The somatic marker hypothesis and the possible functions of the prefrontal cortex. Philosophical Transactions of the Royal Society London B: Biological Science, 351, 1413-1420.

Damasio, A. R., Grabowski, T. J., Bechara, A., Damasio, H., Ponto, L. L. B., Parvizi, J., \& Hichwa, R. D. (2000). Subcortical and cortical brain activity during the feeling of self-generated emotions. Nature Neuroscience, 3, 1049-1056.

Damasio, A. R., Tranel, D., \& Damasio, H. (1990). Individuals with sociopathic behavior caused by frontal damage fail to respond autonomically to social stimuli. Behavioural Brain Research, 41, 81-94.

De Martino, B., Kalisch, R., Rees, G., \& Dolan, R. J. (2009). Enhanced processing of threat stimuli under limited attentional resources. Cerebral Cortex, 19, 127-133.

De Martino, B., Strange, B. A., \& Dolan, R. J. (2008). Noradrenergic neuromodulation of human attention for emotional and neutral stimuli. Psychopharmacology, 197, 127-136.

Dolcos, F., \& Cabeza, R. (2002). Event-related potentials of emotional memory: Encoding pleasant, unpleasant, and neutral pictures. Cognitive, Affective, and Behavioral Neuroscience, 2, 252-263.

Domschke, K., Stevens, S., Pfleiderer, B., \& Gerlach, A. L. (2010). Interoceptive sensitivity in anxiety and anxiety disorders: An overview and integration of neurobiological findings. Clinical Psychology Review, 30, $1-11$.
Donadio, V., Karlsson, T., Elam, M., \& Wallin, B. G. (2002). Interindividual differences in sympathetic and effector responses to arousal in humans. Journal of Physiology-London, 544, 293-302.

Dunn, B. D., Galton, H. C., Morgan, R., Evans, D., Oliver, C., Meyer, M., ... Dalgleish, T. (2010). Listening to your heart: How interoception shapes emotion experience and intuitive decision making. Psychological Science, 21, 1835-1844.

Ehlers, A., \& Breuer, P. (1992). Increased cardiac awareness in panic disorder. Journal of Abnormal Psychology, 101, 371-382.

Ehlers, A., Breuer, P., Dohn, D., \& Fiegenbaum, W. (1995). Heartbeat perception and panic disorder-Possible explanations for discrepant findings. Behaviour Research and Therapy, 33, 69-76.

Elam, M., Yao, T., Svensson, T. H., \& Thoren, P. (1984). Regulation of locus coeruleus neurons and splanchnic, sympathetic nerves by cardiovascular afferents. Brain Research, 290, 281-287.

Gray, M. A., Beacher, F. D., Minati, L., Nagai, Y., Kemp, A. H., Harrison, N. A., \& Critchley, H. D. (2012). Emotional appraisal is influenced by cardiac afferent information. Emotion, 12, 180-191.

Gray, M. A., Minati, L., Paoletti, G., \& Critchley, H. D. (2010). Baroreceptor activation attenuates attentional effects on pain-evoked potentials. Pain, 151, 853-861.

Gray, M. A., Rylander, K., Harrison, N. A., Wallin, B. G., \& Critchley, H. D. (2009). Following one's heart: Cardiac rhythms gate central initiation of sympathetic reflexes. Journal of Neuroscience, 29, 18171825.

Harrison, N. A., Brydon, L., Walker, C., Gray, M. A., Steptoe, A., \& Critchley, H. D. (2009). Inflammation causes mood changes through alterations in subgenual cingulate activity and mesolimbic connectivity. Biological Psychiatry, 66, 407-414.

Harrison, N. A., Brydon, L., Walker, C., Gray, M. A., Steptoe, A., Dolan, R. J., \& Critchley, H. D. (2009). Neural origins of human sickness in interoceptive responses to inflammation. Biological Psychiatry, 66, 415-422.

Heims, H. C., Critchley, H. D., Martin, N. H., Jager, H. R., Mathias, C. J., \& Cipolotti, L. (2006). Cognitive functioning in orthostatic hypotension due to pure autonomic failure. Clinical Autonomic Research, 16, 113120 .

Herbert, B. M., Herbert, C., \& Pollatos, O. (2011). On the relationship between interoceptive awareness and alexithymia: Is interoceptive awareness related to emotional awareness? Journal of Personality, 79, 1149-1175.

Herbert, B. M., Pollatos, O., Flor, H., Enck, P., \& Schandry, R. (2010). Cardiac awareness and autonomic cardiac reactivity during emotional picture viewing and mental stress. Psychophysiology, 47, 342-354.

James, W. (1884). What is an emotion? Mind, 9, 188-205.

James, W. (1890/1950). The Principles of Psychology. Mineola, NY: Dover Publications.

Katkin, E. S. (1985). Blood, sweat, and tears: Individual-differences in autonomic self-perception-Presidential Address, 1984. Psychophysiology, 22, 125-137.

Katkin, E. S., Morell, M. A., Goldband, S., Bernstein, G. L., \& Wise, J. A. (1982). Individual differences in heartbeat discrimination. Psychophysiology, 19, 160-166.

Katkin, E. S., Wiens, S., \& Ohman, A. (2001). Nonconscious fear conditioning, visceral perception, and the development of gut feelings. Psychological Science, 12, 366-370.

Keil, A., \& Ihssen, N. (2004). Identification facilitation for emotionally arousing verbs during the attentional blink. Emotion, 4, 23-35.

Kensinger, E. A., \& Corkin, S. (2003). Memory enhancement for emotional words: Are emotional words more vividly remembered than neutral words? Memory \& Cognition, 31, 1169-1180. 
Khalsa, S. S., Rudrauf, D., Damasio, A. R., Davidson, R. J., Lutz, A., \& Tranel, D. (2008). Interoceptive awareness in experienced meditators. Psychophysiology, 45, 671-677.

Lacey, B. C., \& Lacey, J. I. (1978). 2-way communication between heart and brain-Significance of time within cardiac cycle. American Psychologist, 33, 99-113.

Lange, C. (Ed.). (1885). The emotions. Baltimore, MD: Williams \& Wilkins.

Levinthal, D. J., \& Strick, P. L. (2012). The motor cortex communicates with the kidney. Journal of Neuroscience, 32, 6726-6731.

Liang, K. C., Juler, R. G., \& McGaugh, J. L. (1986). Modulating effects of post-training epinephrine on memory: Involvement of the amygdala noradrenergic system. Brain Research, 368, 125-133.

Liang, K. C., McGaugh, J. L., \& Yao, H. Y. (1990). Involvement of amygdala pathways in the influence of post-training intraamygdala norepinephrine and peripheral epinephrine on memory storage. Brain Research, 508, 225-233.

Ludwickrosenthal, R., \& Neufeld, R. W. J. (1985). Heart beat interoception: A study of individual-differences. International Journal of Psychophysiology, 3, 57-65.

Macmillan, N., \& Creelman, D. (2004). Detection theory: A user's guide. Hove, UK: Psychology Press.

McGaugh, J. L., Cahill, L., \& Roozendaal, B. (1996). Involvement of the amygdala in memory storage: Interaction with other brain systems. Proceedings of the National Academy of Sciences U S A, 93, 1350813514.

McGaugh, J. L., \& Roozendaal, B. (2002). Role of adrenal stress hormones in forming lasting memories in the brain. Current Opinion in Neurobiology, 12, 205-210.

Murase, S., Inui, K., \& Nosaka, S. (1994). Baroreceptor inhibition of the locus-coeruleus noradrenergic neurons. Neuroscience, 61, 635-643.

Nieuwenhuis, S., Gilzenrat, M. S., Holmes, B. D., \& Cohen, J. D. (2005). The role of the locus coeruleus in mediating the attentional blink: A neurocomputational theory. Journal of Experimental Psychology: General, 134, 291-307.

Nogueira, P. J. C., Tomaz, C., \& Williams, C. L. (1994). Contribution of the vagus nerve in mediating the memory-facilitating effects of substance $P$. Behavioural Brain Research, 62, 165-169.
Paulus, M. P., \& Stein, M. B. (2010). Interoception in anxiety and depression. Brain Structure and Function, 214, 451-463.

Pollatos, O., Schandry, R., Auer, D. P., \& Kaufmann, C. (2007). Brain structures mediating cardiovascular arousal and interoceptive awareness. Brain Research, 1141, 178-187.

Schachter, S., \& Singer, J. E. (1962). Cognitive, social, and physiological determinants of emotional state. Psychology Review, 69, 379-399.

Schandry, R., Bestler, M., \& Montoya, P. (1993). On the relation between cardiodynamics and heartbeat perception. Psychophysiology, 30, 467474.

Schwabe, L., \& Wolf, O. T. (2010). Emotional modulation of the attentional blink: Is there an effect of stress? Emotion, 10, 283-288.

Seth, A. K., Suzuki, K., \& Critchley, H. D. (2012). An interoceptive predictive coding model of conscious presence. Frontiers in Psychology, 2, 395-410.

Tsakiris, M., Tajadura-Jimenez, A., \& Costantini, M. (2011). Just a heartbeat away from one's body: Interoceptive sensitivity predicts malleability of body-representations. Proceedings of the Royal Society B-Biological Sciences, 278, 2470-2476.

Vaitl, D. (1996). Interoception. Biological Psychology, 42, 1-27.

Werner, N. S., Jung, K., Duschek, S., \& Schandry, R. (2009). Enhanced cardiac perception is associated with benefits in decision-making. Psychophysiology, 46, 1123-1129.

Werner, N. S., Peres, I., Duschek, S., \& Schandry, R. (2010). Implicit memory for emotional words is modulated by cardiac perception. Biological Psychology, 85, 370-376.

Wiens, S., Mezzacappa, E. S., \& Katkin, E. S. (2000). Heartbeat detection and the experience of emotions. Cognition and Emotion, 14, 417-427.

Williams, C. L., \& McGaugh, J. L. (1993). Reversible lesions of the nucleus of the solitary tract attenuate the memory-modulating effects of posttraining epinephrine. Behavioral Neuroscience, 107, 955-962.

(ReceIVed August 13, 2012; ACCEPTED January 7, 2013) 